\title{
Incorporating single-arm studies in meta-analysis of randomised controlled trials: a simulation study
}

\author{
Janharpreet Singh ${ }^{\text {* }}$ (D), Keith R. Abrams ${ }^{1,2}$ (D) and Sylwia Bujkiewicz ${ }^{1}$ (D)
}

\begin{abstract}
Background: Use of real world data (RWD) from non-randomised studies (e.g. single-arm studies) is increasingly being explored to overcome issues associated with data from randomised controlled trials (RCTs). We aimed to compare methods for pairwise meta-analysis of RCTs and single-arm studies using aggregate data, via a simulation study and application to an illustrative example.

Methods: We considered contrast-based methods proposed by Begg \& Pilote (1991) and arm-based methods by Zhang et al (2019). We performed a simulation study with scenarios varying (i) the proportion of RCTs and single-arm studies in the synthesis (ii) the magnitude of bias, and (iii) between-study heterogeneity. We also applied methods to data from a published health technology assessment (HTA), including three RCTs and 11 single-arm studies.

Results: Our simulation study showed that the hierarchical power and commensurate prior methods by Zhang et al provided a consistent reduction in uncertainty, whilst maintaining over-coverage and small error in scenarios where there was limited RCT data, bias and differences in between-study heterogeneity between the two sets of data. The contrast-based methods provided a reduction in uncertainty, but performed worse in terms of coverage and error, unless there was no marked difference in heterogeneity between the two sets of data.

Conclusions: The hierarchical power and commensurate prior methods provide the most robust approach to synthesising aggregate data from RCTs and single-arm studies, balancing the need to account for bias and differences in between-study heterogeneity, whilst reducing uncertainty in estimates. This work was restricted to considering a pairwise meta-analysis using aggregate data.
\end{abstract}

Keywords: Evidence synthesis, Real world data, Single-arm studies, Bayesian hierarchical methods, Meta-analysis, Arm-based methods

\section{Background}

Health technology assessment (HTA) decision-makers, such as the National Institute for Health and Care Excellence in England and Wales, recommend new health technologies for reimbursement based on cost-effectiveness. They consider the clinical effectiveness of a technology against comparators, estimated by a meta-analysis of

*Correspondence: js929@leicester.ac.uk

${ }^{1}$ Biostatistics Research Group, Department of Health Sciences, University of Leicester, Leicester, UK

Full list of author information is available at the end of the article studies conducted in similar patient populations recording a common outcome measure [1]. A randomised controlled trial (RCT) provides the best evidence of relative effectiveness because random treatment allocation minimises participant selection bias between arms [2]. However, decision-makers may consider observational evidence (e.g. single-arm studies) when, for example, a technology has received accelerated regulatory approval [3]. This suggests a need to develop meta-analysis methods which can combine randomised and non-randomised studies, whilst addressing issues in non-randomised data. 
Bayesian methods provide a flexible approach for combining data from different sources, and can be implemented via Markov chain Monte Carlo (MCMC) sampling which aids probablistic decison-making in HTA [4].

A number of methods have been proposed for pairwise meta-analysis of RCTs and single-arm studies using aggregate data, which make different assumptions regarding data variability. Begg \& Pilote [5] proposed a method under a frequentist framework, which assumes exchangeability for baseline treatment effects and a common relative treatment effect. The method does not distinguish between RCTs and single-arm studies, but can be extended to account for bias in single-arm data. In this context, bias refers to the systematic difference between data from RCTs and single-arm studies. Zhang et al [6] proposed several methods under a Bayesian framework, which assume exchangeability for treatment effects on each arm. The methods distinguish between RCTs and single-arm studies, by assuming correlation between RCT arms and differences in between-study heterogeneity. Some of the methods use single-arm data to inform prior distributions for model parameters. Although Zhang et al performed a simulation study to compare the relative performance between their methods [6], there has been no comparison of these methods with the methods by Begg \& Pilote. Other methods, which are not considered here, use study-matching or individual participant data (IPD) to perform a network meta-analysis (NMA) of RCTs and single-arm studies. Schmitz et al [7] proposed a method using aggregate data on patient characteristics to match single-arm studies with similar patient samples, and perform a NMA of RCTs and the matched studies. Thom et al [8] proposed a method which assumes exchangeability for baseline treatment effects, and uses IPD to adjust for covariates.
In this paper, we focus on the methods proposed by Begg \& Pilote [5] and Zhang et al [6], which combine data from RCTs and single-arm studies at the aggregate level. The two sets of meta-analytic methods are contrastbased and arm-based methods, respectively. We aim to compare both sets of methods to investigate how these different approaches, as well as a number of other specific assumptions, affect their relative performance. We compare the methods in an extensive simulation study, building on the simulation study by Zhang et al [6]. We evaluate performance under a number of scenarios varying the proportion of RCTs and single-arm studies in the synthesis, the magnitude of bias between data from RCTs and single-arm studies, and differences in between-study heterogeneity across RCTs and single-arm studies.

\section{Illustrative example: dataset}

Rheumatoid arthritis (RA) is a chronic auto-immune condition causing joint inflammation, which can be treated by a number of biologic disease-modifying antirheumatic drugs (bDMARDs); adalimumab (ADA), etanercept (ETN), infliximab (IFX), abatacept (ABT), and rituximab (RTX) [9]. The treatment response can be assessed by using the American College of Rheumatology (ACR) response criteria, where ACR20 represents a $20 \%$ improvement in symptoms [10]. Malottki et al assessed the clinical effectiveness of bDMARDs in a HTA [11], identifying three RCTs and 11 single-arm studies for which data were available on the ACR20 outcome. Figure 1 shows a forest plot illustrating the arm-level proportions of ACR20 responders in each study. The plot does not suggest a systematic bias between data from RCTs and single-arm studies on the bDMARD arm.

There are three RCTs in which participants have been assigned a placebo or a bDMARD, and 11 single-arm

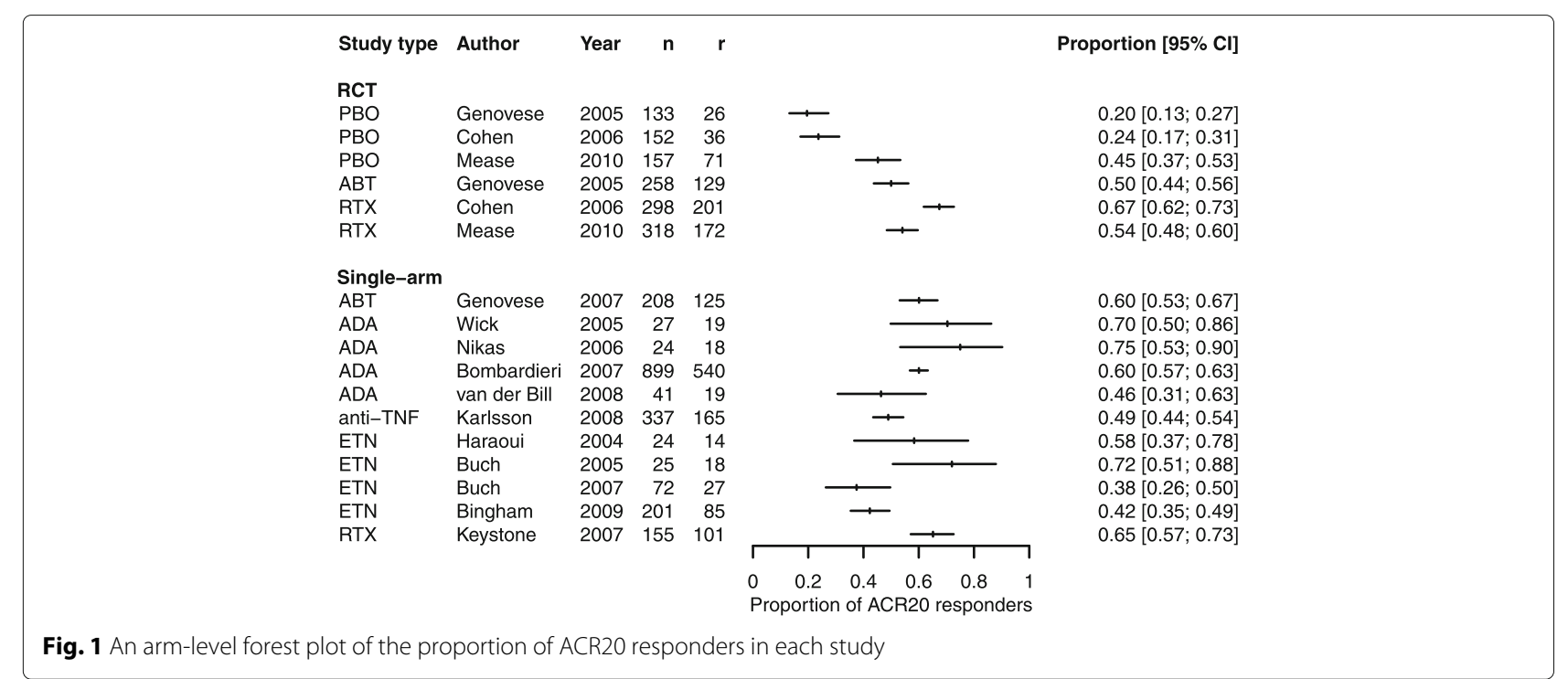


studies in which participants were assigned a bDMARD. We select the three placebo arms as the baseline, so that $\pi_{1}$ represents the marginal response probability for participants assigned a placebo, and $\pi_{2}$ represents the marginal response probability for participants assigned a bDMARD. Thus, the odds ratio represents the increase in odds of achieving a ACR20 response for participants given a bDMARD versus placebo.

\section{Methods}

Methods for meta-analysis of RCTs and single-arm studies using aggregate data

In this section, we describe the methods by Begg \& Pilote [5] and by Zhang et al [6] under a Bayesian framework. Although Begg \& Pilote introduced methods under a frequentist framework, they are adapted here for Bayesian implementation to ensure a fair comparison between both sets of methods. For consistency, and to enable a direct comparison of all methods, we adapt the methods by Begg $\&$ Pilote to a dichotomous outcome. We consider a pairwise meta-analysis, with $n$ RCTs assessing treatments one and two, $m$ single-arm studies assessing treatment one, and $l$ single-arm studies assessing treatment two. We let $i=1, \ldots, n$ index RCTs, $i=n+1, \ldots, n+m$ indexes single-arm studies on arm 1 , and $i=n+m+1, \ldots, n+$ $m+l$ indexes single-arm studies on arm 2 . Here, we describe first the methods introduced by Begg \& Pilote, and then the methods introduced by Zhang et al. The first set of methods parametrise treatment effect contrasts, whilst the latter parametrise treatment effects on each arm. For clarity, we define notation as we introduce each method and attempt to use the original symbols where possible. For the methods by Begg \& Pilote, we begin by describing the original method (BP), and then describe the bias-adjusted (BPbias) and random-effects (BPrandom) methods by showing how they build-on the BP method. For the methods by Zhang et al, we begin by describing the bivariate generalised linear mixed-effects model (BGLMM) I method, and then describe the BGLMM II, hierarchical power prior (HPP) and hierarchical commensurate prior (HCP) methods by showing how they build-on the BGLMM I method. We then describe how marginal response probabilities are calculated for each method.

\section{Begg \& Pilote (BP) original method}

By adapting the method by Begg \& Pilote (BP) to Binomial data, it assumes that in each arm of study $i$ the number of responders follows a Binomial distribution

$$
\begin{aligned}
r_{1 i} & \sim \operatorname{Bin}\left(n_{1 i}, p_{1 i}\right), & & i=1, \ldots, n+m ; \\
r_{2 i} & \sim \operatorname{Bin}\left(n_{2 i}, p_{2 i}\right), & & i=1, \ldots, n ; \quad i=n+m+1, \ldots, n+m+l ;
\end{aligned}
$$

where $n_{1 i}, n_{2 i}$ are the numbers of participants on arms one and two, respectively, and $p_{1 i}, p_{2 i}$ are the response probabilities on arms one and two, respectively. The response probability in each arm is transformed onto the linear predictor scale using a suitable link function $g()$

$$
\begin{aligned}
& g\left(p_{1 i}\right)=\theta_{i}, \quad i=1, \ldots, n+m ; \\
& g\left(p_{2 i}\right)=\theta_{i}+\delta, \quad i=1, \ldots, n ; \quad i=n+m+1, \ldots, n+m+l ;
\end{aligned}
$$

where $\theta_{i}$ represents the baseline treatment effect (i.e. the treatment effect in arm one) in study $i$, and $\delta$ represents the relative treatment effect (i.e. the treatment effect in arm two relative to arm one). Here, the relative treatment effect is assumed to be identical across all studies, whilst the baseline treatment effects are exchangeable (i.e. vary across studies according to a common distribution)

$$
\theta_{i} \sim N\left(\mu, \sigma^{2}\right), \quad i=1, \ldots, n+m+l ;
$$

with mean $\mu$ and standard deviation $\sigma$. Suitably noninformative prior distributions can be placed on $\mu$ and $\sigma$; $\mu \sim N\left(0,10^{5}\right), \sigma \sim \Gamma^{-1}\left(10^{-4}, 10^{-4}\right)$.

\section{Begg \& Pilote method with bias-adjustment (BPbias)}

The bias-adjusted version of the BP method (BPbias) extends BP in Eq. (2) with the additional assumption that single-arm data are systematically biased relative to RCT data

$$
\begin{aligned}
& g\left(p_{1 i}\right)=\theta_{i}+\xi, \quad i=n+1, \ldots, n+m ; \\
& g\left(p_{2 i}\right)=\theta_{i}+\delta+\eta, \quad i=n+m+1, \ldots, n+m+l ;
\end{aligned}
$$

where $\xi$ (for arm one) and $\eta$ (for arm two) represent bias in the single-arm data. The bias is assumed to be common across single-arm studies and suitably non-informative Normal prior distributions can be placed on the bias parameters; $\xi \sim N\left(0,10^{5}\right)$ and $\eta \sim N\left(0,10^{5}\right)$.

\section{Begg \& Pilote method with random effects (BPrandom)}

The BP method with random effects (BPrandom) extends $\mathrm{BP}$ in Eq. (2) by assuming exchangeable relative treatment effects

$$
\begin{aligned}
& g\left(p_{1 i}\right)=\theta_{i}, \quad i=1, \ldots, n+m ; \\
& g\left(p_{2 i}\right)=\theta_{i}+\delta_{i}, \quad i=1, \ldots, n ; \quad i=n+m+1, \ldots, n+m+l ;
\end{aligned}
$$

where $\delta_{i}$ are the relative treatment effects assumed to follow a Normal distribution

$$
\delta_{i} \sim N\left(d, \tau^{2}\right), \quad i=1, \ldots, n ; \quad i=n+m+1, \ldots, n+m+l
$$

with mean $d$ and standard deviation $\tau$. Suitably noninformative prior distributions can be placed on $d$ and $\tau$; $d \sim N\left(0,10^{5}\right), \tau \sim \Gamma^{-1}\left(10^{-4}, 10^{-4}\right)$. 


\section{Bivariate generalised linear mixed effects models (BGLMM) I \& II}

The first method proposed by Zhang et al is bivariate generalised linear mixed-effects model (BGLMM) I, which assumes a Binomial likelihood for the arm-level data as formulated in Eq. (1). In contrast to Begg \& Pilote, Zhang et al model the treatment effect in each arm of study $i$. For RCTs, the method assumes data are correlated between arms

$$
\begin{aligned}
& g\left(p_{1 i}\right)=\mu_{1}+v_{1 i}, \\
& g\left(p_{2 i}\right)=\mu_{2}+v_{2 i}, \\
& \left(v_{1 i}, v_{2 i}\right) \sim N(\mathbf{0}, \Sigma), \quad i=1, \ldots, n ; \\
& \Sigma=\left(\begin{array}{cc}
\sigma_{1}^{2} & \rho \sigma_{1} \sigma_{2} \\
\rho \sigma_{1} \sigma_{2} & \sigma_{2}^{2}
\end{array}\right)
\end{aligned}
$$

where $\mu_{1}$ and $\mu_{2}$ represent the mean treatment effect in each arm, whilst $\left(v_{1 i}, v_{2 i}\right)$ are assumed to follow a bivariate Normal distribution with covariance matrix $\Sigma$, which accounts for between-study heterogeneity across RCTs on each arm and correlation between arms. Non-informative Normal prior distributions can be placed on the mean treatment effects; $\mu_{1} \sim N\left(0,10^{5}\right), \mu_{2} \sim N\left(0,10^{5}\right)$. An inverse-Wishart prior distribution can be placed on the covariance matrix; $\Sigma \sim W^{-1}(R, 2)$, where $R$ is a $2 \times 2$ scale matrix with diagonal elements equal to 1 and offdiagonal elements equal to 0.005 . This prior distribution is weakly informative on both the correlation and standard deviation parameters, but correctly implies that the population-averaged treatment-specific event probabilities range from 0 to 1 . The method assumes the same mean treatment effects $\mu_{1}$ and $\mu_{2}$ for single-arm studies

$$
\begin{array}{lll}
g\left(p_{1 i}\right)=\mu_{1}+v_{3 i}, & v_{3 i} \sim N\left(0, \sigma_{3}^{2}\right) & i=n+1, \ldots, n+m ; \\
g\left(p_{2 i}\right)=\mu_{2}+v_{4 i}, & v_{4 i} \sim N\left(0, \sigma_{4}^{2}\right) & i=n+m+1, \ldots, n+m+l ;
\end{array}
$$

where $v_{3 i}$ and $v_{4 i}$ are each assumed to follow a univariate Normal distribution to account for the between-study heterogeneity across single-arm studies on each arm. Similar to Zhang et al, we place inverse-Gamma prior distributions on the standard deviation parameters; $\sigma_{3} \sim$ $\Gamma^{-1}\left(10^{-4}, 10^{-4}\right)$ and $\sigma_{4} \sim \Gamma^{-1}\left(10^{-4}, 10^{-4}\right)$.

The BGLMM I method can be modified in Eq. (8) to assume different mean treatment effects $\mu_{3}$ and $\mu_{4}$ for single-arm studies

$$
\begin{array}{lll}
g\left(p_{1 i}\right)=\mu_{3}+v_{3 i}, & v_{3 i} \sim N\left(0, \sigma_{3}^{2}\right) & i=n+1, \ldots, n+m ; \\
g\left(p_{2 i}\right)=\mu_{4}+v_{4 i}, & v_{4 i} \sim N\left(0, \sigma_{4}^{2}\right) & i=n+m+1, \ldots, n+m+l ;
\end{array}
$$

Non-informative Normal prior distributions can be placed on the mean treatment effects; $\mu_{3} \sim N\left(0,10^{5}\right)$, $\mu_{4} \sim N\left(0,10^{5}\right)$, which can themselves be applied to inform prior distributions for $\mu_{1}$ and $\mu_{2}$ in a two-step method. First, the model specified in Eq. (9) is fit to the single-arm data to estimate posterior distributions for $\mu_{3}$ and $\mu_{4}$, from which posterior median and standard deviation estimates are obtained. Then, the model specified in Eq. (7) is fit to the RCT data, with informative prior distributions (based on the extracted estimates) placed on the mean treatment effects; $\mu_{1} \sim N\left(\hat{\mu_{3}},{\hat{\tau_{1}}}^{2}\right)$, $\mu_{2} \sim N\left(\hat{\mu_{4}},{\hat{\tau_{2}}}^{2}\right)$. This modified-version of the BGLMM I method is labelled BGLMM II.

\section{Hierarchical power prior (HPP)}

The hierarchical power prior (HPP) method extends the BGLMM I method in Eq. (1) by raising the likelihood functions $L\left(p_{1 i}\right)$ and $L\left(p_{2 i}\right)$ for the single-arm studies to a power between zero and one

$L\left(p_{1 i}\right)=\left(p_{1 i}^{r_{1 i}}\left(1-p_{1 i}\right)^{n_{1 i}-r_{1 i}}\right)^{\alpha_{1}}, \quad i=n+1, \ldots, n+m ;$

$L\left(p_{2 i}\right)=\left(p_{2 i}^{r_{2 i}}\left(1-p_{2 i}\right)^{n_{2 i}-r_{2 i}}\right)^{\alpha_{2}}, \quad i=n+m+1, \ldots, n+m+l$;

where $\alpha_{1}$ and $\alpha_{2}$ represent the power parameters for each arm. To allow flexibility in down-weighting the single-arm data, Beta prior distributions can be placed on the power parameters; $\alpha_{1} \sim \beta(10,1), \alpha_{2} \sim \beta(10,1)$. A $\beta(10,1)$ prior has mean 0.91 and a $95 \%$ credible interval ranging from 0.69 to 0.99 , which indicates a moderate-to-strong similarity between single-arm studies and RCTs, and provides a modest down-weighting [6].

\section{Hierarchical commensurate prior (HCP)}

The hierarchical commensurate prior (HCP) method assumes different mean treatment effects for RCTs and single-arm studies (described by Eqs. (7) and (9)), and places Normal prior distributions on $\mu_{1}$ and $\mu_{2}$ informed by the single-arm data;

$$
\begin{aligned}
& \mu_{1} \sim N\left(\mu_{3}, \frac{1}{\tau_{1}^{2}}\right) \\
& \mu_{2} \sim N\left(\mu_{4}, \frac{1}{\tau_{2}^{2}}\right)
\end{aligned}
$$

where $\tau_{1}$ and $\tau_{2}$ are commensurability parameters representing agreement between data from RCTs and singlearm studies. Similar to Zhang et al, we place Gamma prior distributions on each parameter; $\tau_{1} \sim \Gamma\left(10^{-3}, 10^{-3}\right)$, $\tau_{2} \sim \Gamma\left(10^{-3}, 10^{-3}\right)$. For small parameter values, the variance of the single-arm data is inflated and the contribution to $\mu_{1}$ and $\mu_{2}$ is down-weighted. As parameter values approach zero, only RCT data contribute in estimating $\mu_{1}$ and $\mu_{2}$, whilst single-arm data are ignored. As the parameter values approach infinity, data from RCTs and single-arm studies contribute equally in estimating $\mu_{1}$ and $\mu_{2}$. 


\section{Marginal response probabilities}

The methods described above for a dichotomous outcome model the response probability in each arm, based on the numbers of participants and responders (described by Eq. (1)), and use a link function $g()$ to transform the response probability onto the linear predictor scale where treatment effects are additive. A logit or probit link function can be used for meta-analysis with Binomial data [4], although the logit link is often favoured in the published literature as the relative treatment effects are easier to interpret on the log odds ratio scale. The methods proposed by Zhang et al do not parametrise relative treatment effects, and instead they recommend using the probit link $\Phi^{-1}()$ and then calculating the marginal response probability in each arm

$$
\begin{aligned}
& \pi_{1}=\Phi\left(\frac{\mu_{1}}{\sqrt{1+\sigma_{1}^{2}}}\right) \\
& \pi_{2}=\Phi\left(\frac{\mu_{2}}{\sqrt{1+\sigma_{2}^{2}}}\right)
\end{aligned}
$$

where $\Phi$ is the cumulative distribution function for the standard Normal distribution. The marginal response probabilities can be used to calculate a marginal odds ratio $O R_{21}=\pi_{2}\left(1-\pi_{1}\right) / \pi_{1}\left(1-\pi_{2}\right)$. We implement the methods proposed by Begg \& Pilote using a probit link to allow a direct comparison of all methods. For the BP and BPbias methods, we obtain the marginal response probabilities using Eq. (13)

$$
\begin{aligned}
& \pi_{1}=\Phi\left(\frac{\mu}{\sqrt{1+\sigma^{2}}}\right) \\
& \pi_{2}=\Phi\left(\frac{\mu+\delta}{\sqrt{1+\sigma^{2}}}\right)
\end{aligned}
$$

and for the BPrandom method using Eq. (14)

$$
\begin{aligned}
& \pi_{1}=\Phi\left(\frac{\mu}{\sqrt{1+\sigma^{2}}}\right) \\
& \pi_{2}=\Phi\left(\frac{\mu+d}{\sqrt{1+(\sigma+\tau)^{2}}}\right)
\end{aligned}
$$

\section{Summary of methods}

In this section, we have described the details of each method (including suitable prior distributions) and the corresponding marginal response probabilities (WinBUGS code used to fit each of the methods is provided in Appendix D). We note that the methods proposed by Zhang et al reduce to the model described by Eq. (7) when applied to RCT data only $(i=1, \ldots, n)$, which we label BGLMM*. Similarly, the BP and BPbias methods reduce to the model described by Eqs. (2) and (3) when applied to
RCT data only, which we label BP*. We label the BPrandom method applied to RCT data only as BPrandom*.

\section{Simulation study: methods}

In this section, we report aims, data-generation methods, estimands, methods, and performance measures for the simulation study, as recommended by Morris et al [12]. The simulation study aimed to compare the performance of the methods described previously, under a number of scenarios varying the proportion of RCTs and single-arm studies in the synthesis, the magnitude of bias between data from RCTs and single-arm studies, and differences in between-study heterogeneity across RCTs and singlearm studies. We aimed to build-on the simulation study performed by Zhang et al [6], where the estimands were the marginal response probability in each arm $\pi_{1}$ and $\pi_{2}$. We evaluated performance by calculating coverage, meansquare error (MSE), and mean change in 95\% credible interval length (CrIL). The latter measures the average change in CrIL when a method is applied to RCT and single-arm data versus $R C T$ data only. The methods were implemented via MCMC sampling in the WinBUGS software, using a burn-in of 20,000 iterations and 100,000 iterations for posterior estimation [13].

\section{Data-generation methods}

As in Zhang et al [6], we let $n$ represent the number of RCTs assessing treatments one and two, $m$ - the number of single-arm studies assessing treatment one, and $l$ - the number of single-arm studies assessing treatment two. We let $i$ denote study, and set the total number of studies in a dataset $n+m+l=30$. The data were simulated basedon the BGLMM I method, modified to assume bias in the single-arm data. The steps taken to simulate a dataset were as follows. For RCT data, we specified values for the between-study heterogeneity on each arm $\left(\sigma_{1}\right.$ and $\left.\sigma_{2}\right)$ and correlation between arms $(\rho)$ to obtain the covariance matrix $(\Sigma)$ to simulate $v_{1 i}$ and $v_{2 i}$

$$
\begin{aligned}
& \Sigma=\left(\begin{array}{cc}
\sigma_{1}^{2} & \rho \sigma_{1} \sigma_{2} \\
\rho \sigma_{1} \sigma_{2} & \sigma_{2}^{2}
\end{array}\right) \\
& \left(v_{1 i}, v_{2 i}\right) \sim N(\mathbf{0}, \Sigma), \quad i=1, \ldots, n ;
\end{aligned}
$$

We assigned values to the mean treatment effect in each arm $\left(\mu_{1}\right.$ and $\left.\mu_{2}\right)$, and applied the simulated $v_{1 i}$ and $v_{2 i}$ to obtain response probabilities on each arm

$$
\begin{aligned}
& p_{1 i}=\Phi\left(\mu_{1}+v_{1 i}\right), \\
& p_{2 i}=\Phi\left(\mu_{2}+v_{2 i}\right), \quad i=1, \ldots, n ;
\end{aligned}
$$

where $\Phi$ is the cumulative distribution function for the standard Normal distribution. We set the number of participants to 100 in each arm of study $i$, and applied the response probabilities $\left(p_{1 i}\right.$ and $\left.p_{2 i}\right)$ to sample the number of responders $\left(r_{1 i}\right.$ and $\left.r_{2 i}\right)$ from a Binomial distribution 


$$
\begin{aligned}
r_{1 i} & \sim \operatorname{Bin}\left(100, p_{1 i}\right), \\
r_{2 i} & \sim \operatorname{Bin}\left(100, p_{2 i}\right), \quad i=1, \ldots, n ;
\end{aligned}
$$

For single-arm data, we specified values for the betweenstudy heterogeneity on each arm $\left(\sigma_{3}\right.$ and $\left.\sigma_{4}\right)$ to simulate $v_{3 i}$ and $v_{4 i}$

$$
\begin{aligned}
& v_{3 i} \sim N\left(0, \sigma_{3}^{2}\right) \quad i=n+1, \ldots, n+m ; \\
& v_{4 i} \sim\left(0, \sigma_{4}^{2}\right) \quad i=n+m+1, \ldots, n+m+l ;
\end{aligned}
$$

We defined values for the bias in each arm $(\xi$ and $\eta)$, and applied the simulated $v_{3 i}$ and $v_{4 i}$ together with the mean treatment effects $\left(\mu_{1}\right.$ and $\left.\mu_{2}\right)$, to obtain the response probabilities on each arm

$$
\begin{array}{ll}
p_{1 i}=\Phi\left(\mu_{1}+v_{3 i}+\xi\right), & i=n+1, \ldots, n+m ; \\
p_{2 i}=\Phi\left(\mu_{2}+v_{4 i}+\eta\right), & i=n+m+1, \ldots, n+m+l ;
\end{array}
$$

We applied the response probabilities $\left(p_{1 i}\right.$ and $\left.p_{2 i}\right)$ to sample the numbers of responders $\left(r_{1 i}\right.$ and $\left.r_{2 i}\right)$ from a Binomial distribution

$$
\begin{array}{rlrl}
r_{1 i} & \sim \operatorname{Bin}\left(100, p_{1 i}\right), & i & =n+1, \ldots, n+m ; \\
r_{2 i} \sim \operatorname{Bin}\left(100, p_{2 i}\right), & & i=n+m+1, \ldots, n+m+l ;
\end{array}
$$

The data were simulated under a number of scenarios adapted from scenario 1 (S1), where the number of RCTs $n=15$ and the number of single-arm studies on each arm $m=10$ and $l=5$. The magnitude of bias for singlearm studies $\xi=0.2$ and $\eta=0.4$, and between-study heterogeneity parameters $\sigma_{1}=0.6, \sigma_{2}=0.7, \sigma_{3}=0.8$, $\sigma_{4}=1$. Due to lack of randomisation, single-arm data are at a higher risk of bias compared to randomised data, so we assume a systematic difference (i.e. parameters $\xi=0.2$ and $\eta=0.4$ ) and larger between-study heterogeneity (i.e. parameters $\sigma_{3}=0.8$ and $\left.\sigma_{4}=1.0\right)$. For all scenarios, the mean treatment effects were set to $\mu_{1}=0.4$ and $\mu_{2}=1.1$, and correlation was $\rho=0.6$. We arrange the scenarios into four groups, where in each group the scenarios focus on varying a common set of parameter values. In group one, S1-5, the number of RCTs gradually decreases (from $n=15$ to $n=1$ ). This was intended to clearly demonstrate the performance of the methods in scenarios where there is little randomised evidence (i.e. S4 and S5, where $n=3$ and $n=1$ ) compared to scenarios where there is relatively substantial randomised evidence (i.e. $\mathrm{S} 1$, where $n=15$ ). In group two, [S6, S1, S7-9], the bias gradually increases (from $\xi=0, \eta=0$ to $\xi=0.8, \eta=1$ ). In group three, [S10-12, S6], the between-study heterogeneity for single-arm data gradually increases (from $\sigma_{3}=0.1, \sigma_{4}=$ 0.3 to $\left.\sigma_{3}=0.8, \sigma_{4}=1\right)$, with zero bias $(\xi=0, \eta=0)$. In group four, [S13-15, S1], the between-study heterogeneity for the single-arm data gradually increases (from $\sigma_{3}=0.1, \sigma_{4}=0.3$ to $\sigma_{3}=0.8, \sigma_{4}=1$ ), with non-zero bias $(\xi=0.2, \eta=0.4)$. A full description of the parameter values specified in each scenario is provided in Table A.1 (Appendix A).

\section{Results}

\section{Illustrative example: results}

Table 1 presents posterior median estimates (and 95\% credible intervals) for $\pi_{1}, \pi_{2}$, and the marginal odds ratio. The results are presented separately for analysis of $R C T$ and single-arm data versus analysis of RCT data only. For the latter analysis, a random-effects meta-analysis (REMA) [14] and fixed-effect meta-analysis (FEMA) were also implemented. The odds ratio estimates range from 2.53 to 3.4 , suggesting participants assigned a bDMARD versus placebo were more than twice as likely to achieve an ACR20 response. However, only the contrast-based methods show CrIs greater than one. There is a reduction in

Table 1 Posterior median estimates and 95\% credible intervals from application to illustrative example

\begin{tabular}{lllll}
\hline Method & $\pi_{\mathbf{1}}$ & $\boldsymbol{\pi}_{\mathbf{2}}$ & OR & DIC \\
\hline BGLMM1 & $0.32(0.14,0.59)$ & $0.55(0.49,0.62)$ & $2.65(0.84,7.68)$ & $104.10(94.93,118.30)$ \\
BGLMM2 & $0.33(0.15,0.62)$ & $0.55(0.49,0.62)$ & $2.53(0.74,7.31)$ & \\
HPP & $0.32(0.13,0.60)$ & $0.56(0.40,0.72)$ & $2.73(0.74,10.64)$ & $104.20(95.07,118.40)$ \\
HCP & $0.32(0.13,0.64)$ & $0.56(0.35,0.72)$ & $2.60(0.61,9.38)$ & $131.40(122.70,144.90)$ \\
BP & $0.30(0.23,0.37)$ & $0.56(0.51,0.62)$ & $3.06(2.42,3.89)$ & $131.10(122.60,144.90)$ \\
BPbias & $0.30(0.20,0.44)$ & $0.57(0.45,0.69)$ & $3.06(2.41,3.93)$ & $103.90(94.82,118.40)$ \\
BPrandom & $0.30(0.15,0.53)$ & $0.55(0.29,0.77)$ & $2.74(1.05,6.78)$ & 10
\end{tabular}

\section{RCT data only}

BGLMM*

BP*

BPrandom*

REMA

FEMA
$0.32(0.13,0.61)$

$0.30(0.19,0.49)$

$0.31(0.15,0.58)$
$0.56(0.28,0.78)$

$0.57(0.40,0.71)$

$0.56(0.26,0.81)$
$2.64(0.51,13.32)$

$3.00(2.00,3.89)$

$2.76(0.95,7.85)$

$3.40(0.82,14.02)$

$3.18(2.49,4.08)$
$39.08(34.99,48.09)$

$65.84(62.91,73.80)$

$39.59(35.11,49.61)$

$39.17(35.02,48.34)$

$65.95(63.07,73.78)$ 
uncertainty when methods are applied to include singlearm studies in the synthesis, and the arm-based methods show a greater reduction in CrIL for the odds ratio (between 23-49\%) compared to the contrast-based methods (between 17-22\%). Table 1 includes estimates for the deviance information criterion (DIC), which provides a measure of model fit whilst penalising model complexity [15]. The BP method has the highest DIC value and is the simplest method in terms of model parameters.

\section{Simulation study: results}

In this section, we present the simulation study results for each method in terms of coverage, MSE and mean change in CrIL. We illustrate the results for each scenario group with a line plot of the performance measures for each estimand.

\section{Scenarios S1-5}

Across scenarios S1-5, the proportion of RCTs gradually decreases (from $n=15$ to $n=1$ ), whilst the total number of studies remains fixed $(n+m+l=30)$. The results for these scenarios are presented in Fig. 2. The HPP and HCP methods, which both down-weight the single-arm data, perform relatively strongly with overcoverage (i.e. coverage above the nominal value 0.95 ) and small MSE for both estimands. This suggests that downweighting the single-arm data can mitigate the effect of bias. The BPbias method, which includes a parameter on each arm to account for bias, performs strongly in S1-2 where there is a significant number of RCTs $(n=15$ and $n=12$ ). However, its performance drops-off and MSE is much larger in S4-5 where there are few RCTs $(n=3$ and $n=1$ ). This suggests that it requires a significant number of both RCTs and single-arm studies to estimate bias. The BP method is naive to study-design, and shows under-coverage for all scenarios, which worsens as the proportion of RCTs decreases. All methods, aside from BPbias, provide a reduction in uncertainty when including versus excluding single-arm data.

In scenarios S1-5, data were simulated using the BGLMM I method, potentially favouring arm-based methods over contrast-based methods. We performed a sensitivity analysis to further explore this, where data were simulated using the BPrandom method. We label these scenarios $\mathrm{S}^{*}-5^{*}$, and the results are presented in Figure B.1 (Appendix B). The HCP and HPP methods performed strongly across S1-5, and maintain their performance in $S 1^{*}-5^{*}$ with over-coverage and relatively small MSE. The performance of BPbias in $\mathrm{S} 1^{*}-5^{*}$ mirrors its performance in S1-5, with a significant decrease in coverage and increase in MSE occurring in $\mathrm{S}^{*}$. The $\mathrm{BP}$ and BPrandom methods show a reduction in undercoverage and MSE, perhaps because their assumptions are now better aligned with the data-generating method (e.g. common between-study heterogeneity across all studies). In contrast, the BGLMM I and II methods show a reduction in coverage and a small increase in MSE, because their assumptions are not as aligned to the datagenerating method. The impact of between-study heterogeneity is explored further in scenarios [S10-12, S6] and [S13-15, S6].

\section{Scenarios [S6, S1, S7-9]}

Across scenarios [S6, S1, S7-9], the magnitude of bias in each arm gradually increases (from $\xi=0, \eta=0$ to $\xi=0.8, \eta=1)$. The results for these scenarios are presented in Fig. 3. The BPbias method shows consistent over-coverage and small MSE, but does not offer any reduction in uncertainty when including single-arm studies. The HCP and HPP methods maintain coverage close to the nominal value and small MSE, whilst offering a consistent reduction in uncertainty. They only show a drop in performance in S9, where there is relatively large bias in single-arm data. The BP and BPrandom methods are naive to study-design and show reduction in uncertainty, but a steep decrease in coverage and increase in MSE as the bias is increased. The drop in performance is worse for $\pi_{1}$ than $\pi_{2}$, perhaps because there are more single-arm studies on arm one $(m=10)$ than arm two $(l=5)$. The BGLMM I and II methods do not account for bias in the single-arm data, but show a more gradual decrease in coverage and increase in MSE.

\section{Scenarios $[S 10-12,56]$}

Across scenarios [S10-12, S6], between-study heterogeneity in single-arm studies on each arm gradually increases (from $\sigma_{3}=0.1, \sigma_{4}=0.3$ to $\sigma_{3}=0.8, \sigma_{4}=1$ ) but remains fixed in RCTs $\left(\sigma_{1}=0.6, \sigma_{2}=0.7\right)$, and there is zero bias $(\xi=0, \eta=0)$. Figure 4 presents the results for these scenarios. The BPbias method shows significant under-coverage and large MSE in S10 where the single-arm studies have much lower between-study heterogeneity compared to RCTs, but still provides some reduction in uncertainty. The BP and BPrandom methods show less under-coverage and much smaller MSE, whilst providing a greater reduction in uncertainty. The BGLMM I and II methods show over-coverage and little MSE, whilst providing the greatest reduction in uncertainty. As the between-study heterogeneity is increased, all methods show a decrease for the reduction in uncertainty, but the HCP and HPP methods are impacted the least.

\section{Scenarios [S13-15, S1]}

In contrast to [S10-12, S6], scenarios [S13-15, S1] assume non-zero bias in the single-arm data $(\xi=0.2, \eta=0.4)$, and the results are presented in Fig. 5. In S13, where single-arm data is much less uncertain than the RCT data, the BGLMM I and II methods show a significant 


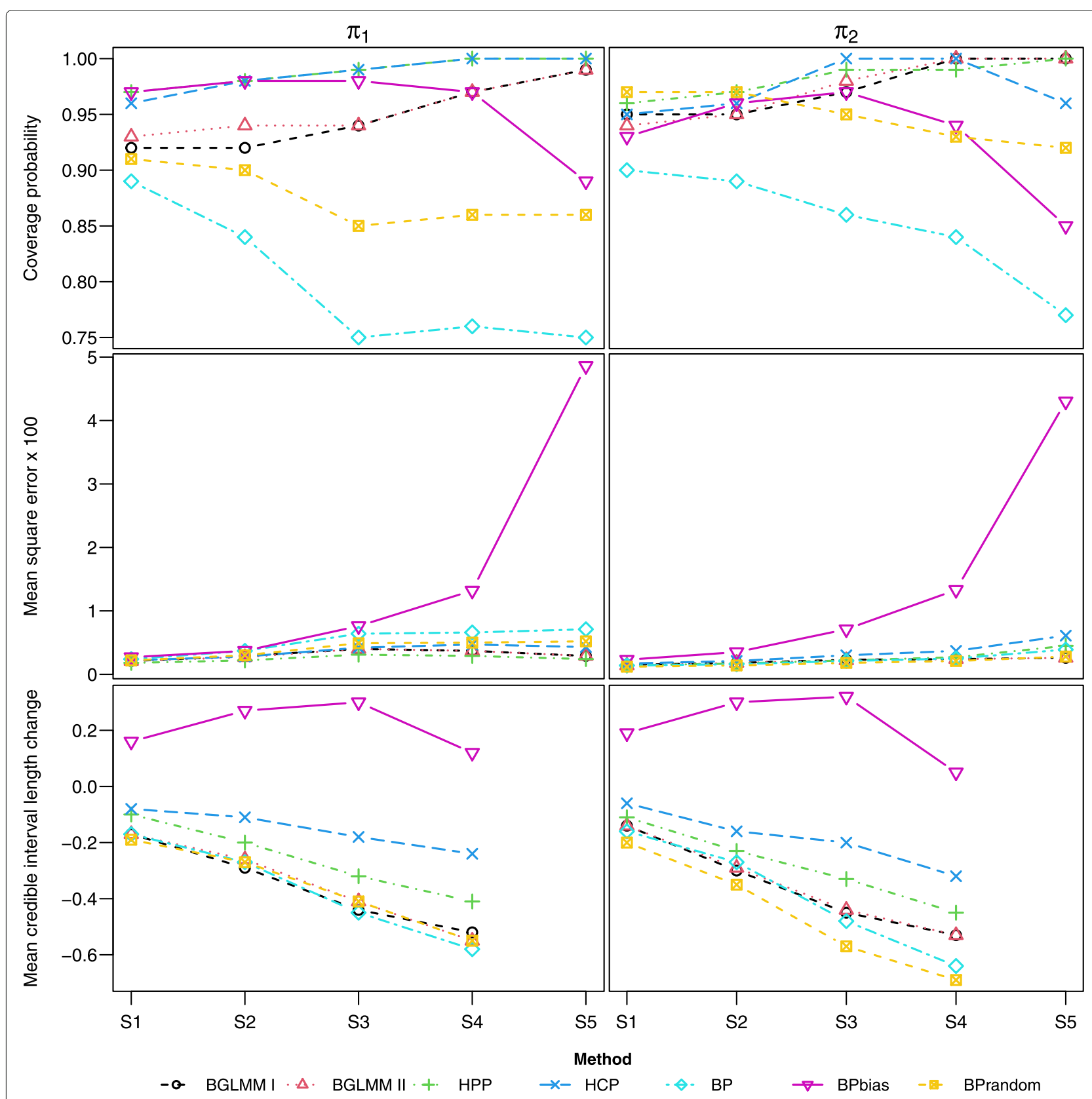

Fig. 2 Coverage, MSE, and mean change in CrlL for each estimand, across scenarios S1-5 where the number of RCTs is gradually decreased

reduction in uncertainty but large under-coverage and significant MSE. In comparison, the BP and BPrandom methods provide a more modest reduction in uncertainty but better coverage, although the BPrandom method shows large MSE. The BPbias method shows improvement compared to $\mathrm{S} 10$, but offers only a modest reduction in uncertainty which diminishes in [S14-15, S1]. The HCP and HPP methods, which down-weight single-arm data, show over-coverage and small MSE across the scenarios whilst maintaining a reduction in uncertainty.

\section{Discussion}

In this paper, we aimed to compare methods proposed by Begg \& Pilote and Zhang et al, for pairwise meta-analysis combining data from RCTs and single-arm studies using aggregate data. Based on our simulation study, we conclude that the HCP and HPP methods provide a consistent reduction in uncertainty when including single-arm data, whilst remaining robust to limited RCT data, bias, and differences in between-study heterogeneity across the two sets of data. Both methods achieve this by down- 


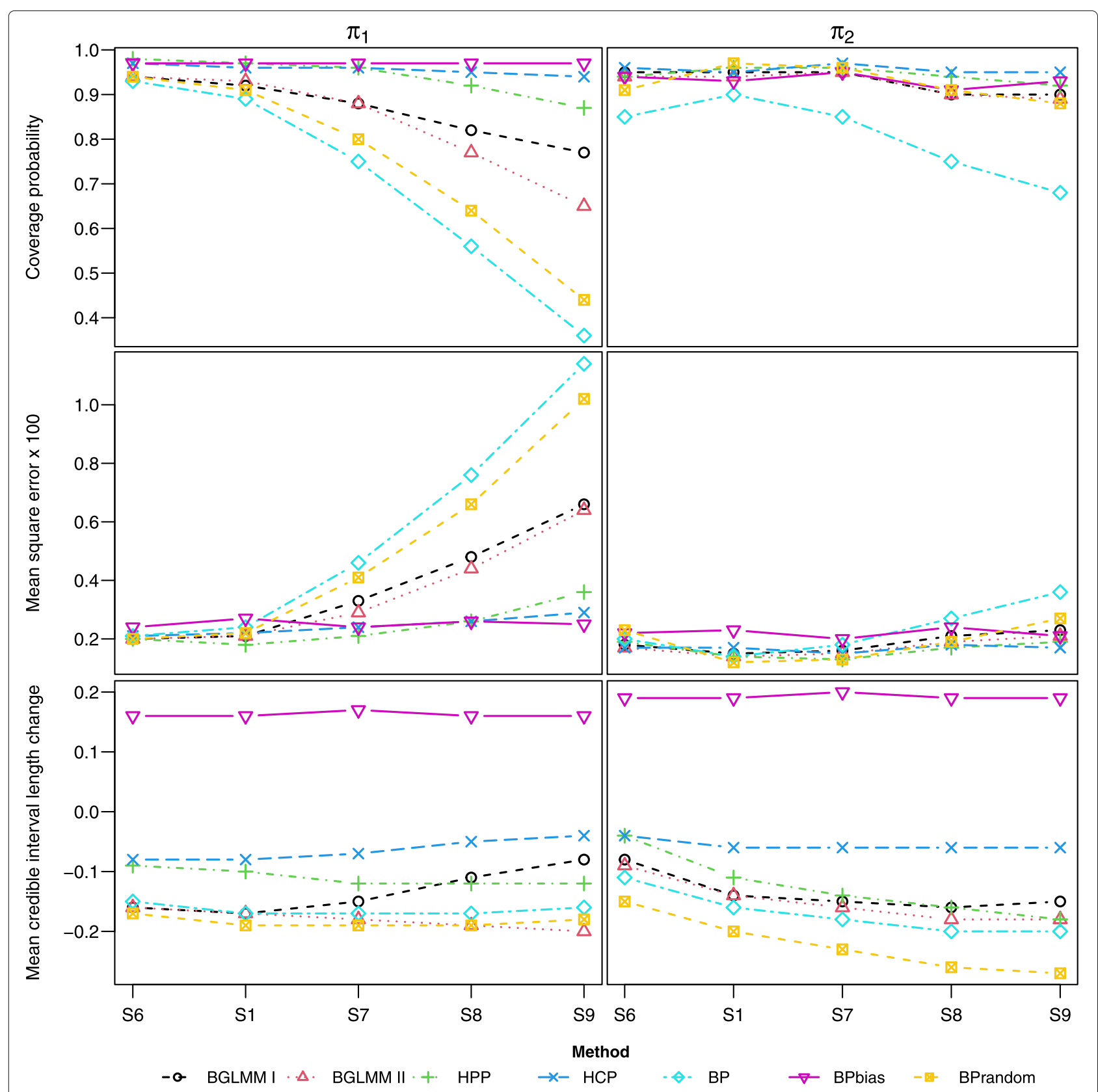

Fig. 3 Coverage, MSE, and mean CrlL change for each estimand, across scenarios [S6, S1, S7-9] where bias in each arm gradually increases

weighting the single-arm data, HPP through specification of a prior distribution, and HCP through estimating disagreement between the data from RCTs and single-arm studies. The BPbias method offers a simpler approach to mitigating bias, but requires a significant proportion of RCTs and single-arm studies in the synthesis. The BGLMM I and II methods provide a reduction in uncertainty, contingent upon little or no bias. Through our analysis of an illustrative example, we have shown that the methods can be used to combine data from RCTs and single-arm studies to achieve a significant reduction in uncertainty, compared to traditional meta-analysis of RCTs alone. We list below key recommendations in applying the methods for synthesis of RCTs and single-arm studies.

\section{Key recommendations:}

- The BP method is a parsimonious approach offering significant reduction in uncertainty (compared to the analysis of RCT data alone), when there is little or no bias and differences in between-study heterogeneity between the two types of data. 


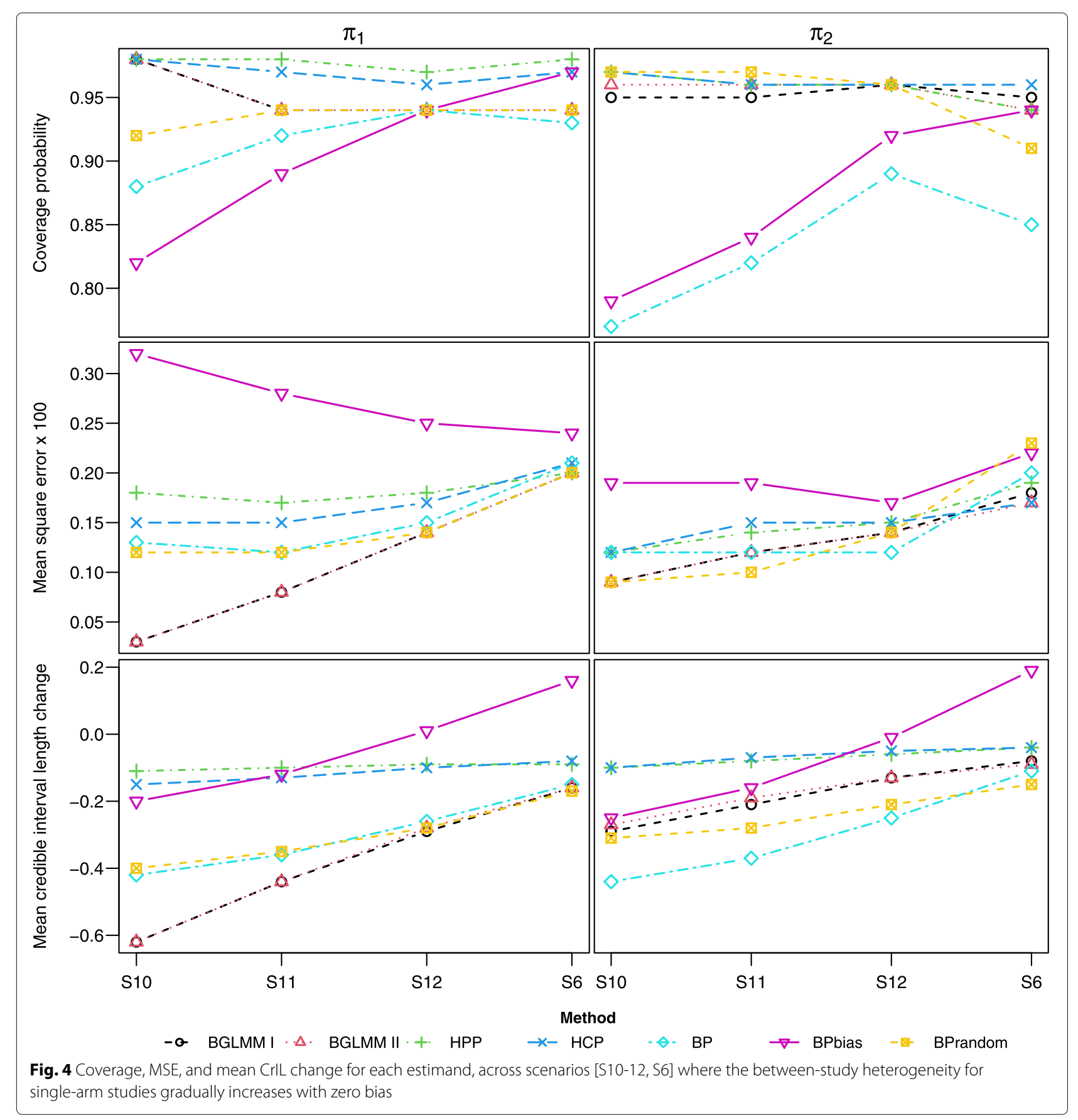

- The BGLMM I and II methods provide a significant reduction in uncertainty whilst accounting for differences in between-study heterogeneity, when there is little or no bias.

- The HPP method allows for down-weighting singlearm data, and remains robust to limited RCT data and bias whilst providing a reduction in uncertainty.

- The HCP method provides a consistent reduction in uncertainty whilst accounting for disagreement between RCT and single-arm data, and also remains robust to limited RCT data.
In a traditional meta-analysis of RCTs aiming to estimate a pooled relative treatment effect [16], baseline treatment effects are allowed to vary independently to preserve randomisation in each arm and minimise bias. There has been discussion in the literature regarding arm-based and contrast-based approaches to meta-analysis. Hong et al have suggested arm-based methods can minimise bias when data are assumed to be missing in a particular arm [17]. In response, Dias \& Ades have argued that arm-based models actually increase bias since they do not preserve randomisation [18]. A further exploration of constrast- 


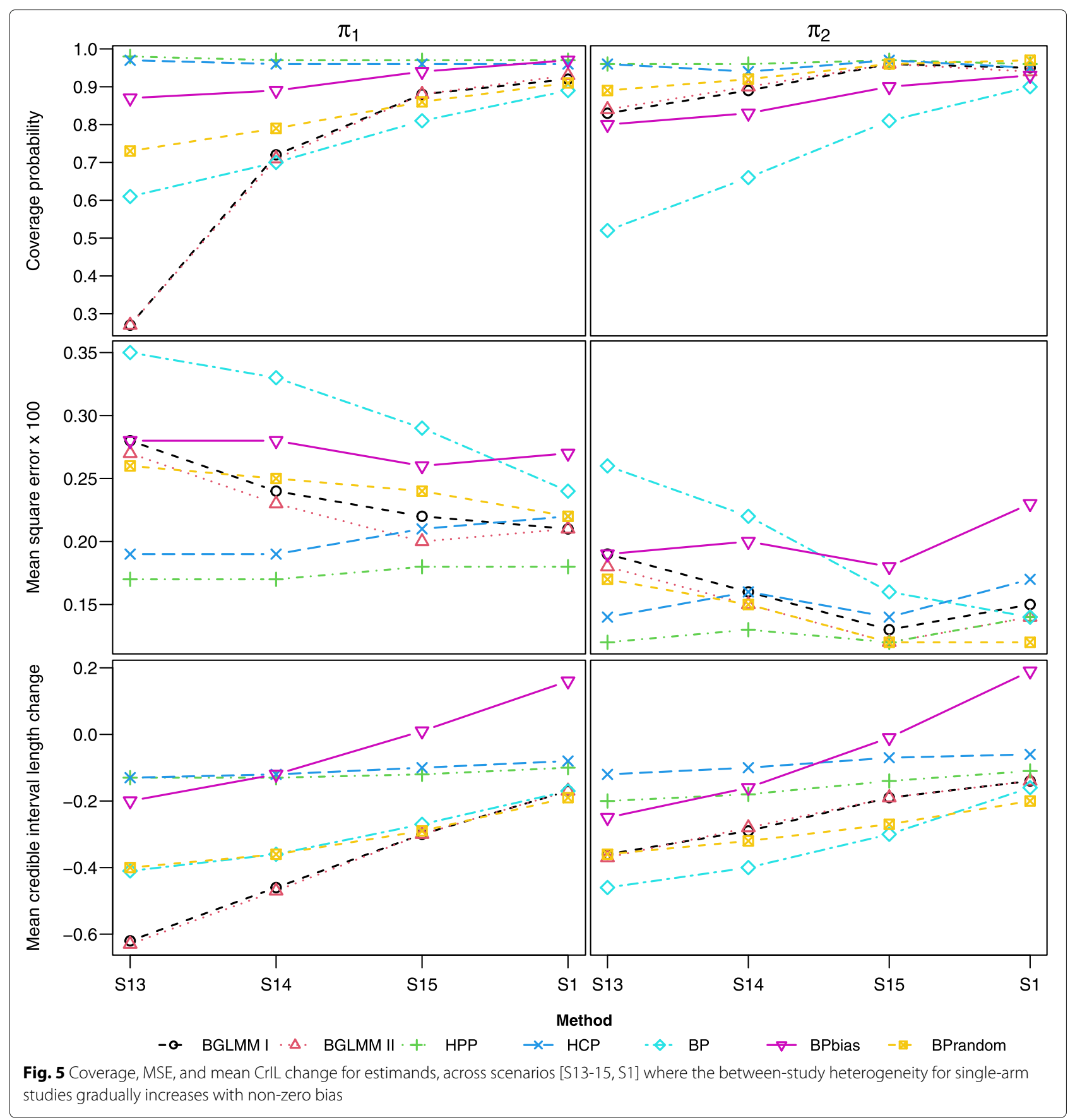

based and arm-based models has been performed by White et al considering a NMA context [19]. The traditional meta-analysis approach is not feasible when seeking to combine RCTs and single-arm studies, because the single-arm studies lack a comparator arm to estimate a relative treatment effect. Consequently, exchangeability must be assumed on at least one arm to incorporate the single-arm studies. The methods by Begg \& Pilote assume exchangeability on the designated baseline arm, whilst those by Zhang et al assume exchangeability on both arms.
Thus, it may be beneficial to perform a sensitivity analysis using more than one method. Decision-makers can then consider the benefits offered by including the single-arm studies (e.g. reduction in uncertainty) versus the potential penalties (e.g. increased risk of bias), and whether the penalties have been mitigated by applying a suitable method.

Aside from application in HTA, the methods assessed here can also be useful in clinical settings, for instance, in early-phase cancer research. Phase II cancer trials assess 
treatment efficacy via randomised-controlled or singlearm study designs, where only the latter may be ethical for rare cancers [20]. Consequently, there may be data available from both RCTs and single-arm studies, which need to be synthesised to determine the feasibility of a phase III trial [21]. Thus, further methodological development for performing a meta-analysis (or NMA [22]) to combine data from different study designs is required.

In this paper, we have considered the case where only aggregate data are available, which limits the methods to rudimentary bias-adjustment. The methods, however, can be very useful in situations when there are no IPD available and RCT data are limited. Further research is required to explore methods adjusting for bias which is variable across studies, to account for differences in risk of bias due to study setting (e.g. single-centre versus multi-centre single-arm studies). When IPD are available, a more detailed adjustment for potential biases can be carried out, as there are a number of approaches available that can be applied to mitigate confounding when estimating causal treatment effects [23]. For example, availability of IPD allows for enhancing approaches for meta-regression, which is recommended to explore bias and heterogeneity in a synthesis of evidence [24]. Furthermore, we did not consider the methods recently proposed by Schmitz et al [7], which incorporate single-arm studies in NMA of RCTs. Although proposed under a NMA context, they can be adapted for pairwise meta-analysis. However, after matching single-arm studies based on covariate information, the models used to synthesise data are only applicable to two-arm studies. Including those methods in this simulation study would also require specifying a model from which to simulate data on covariates. Thus, the simulation study was restricted to the methods by Begg \& Pilote and Zhang et al.

\section{Conclusions}

We have performed an extensive comparison of methods proposed by Begg \& Pilote and Zhang et al, for pairwise meta-analysis combining data from RCTs and single-arm studies using aggregate data. We conclude that those methods by Zhang et al (HCP and HPP), which use the single-arm data to define prior distribution for model parameters, provide a consistent reduction in uncertainty when including single-arm data, whilst remaining robust to data variability. The other methods considered here perform worse when there is limited RCT data (BPbias), significant bias (BGLMM I \& II), and differences in between-studies heterogeneity across the two sets of data (BP and BPrandom). We hope this study is informative for researchers seeking to perform a pairwise meta-analysis of RCTs and single-arm studies using aggregate data. We have described the existing methods in detail under a Bayesian framework, and the methods' advantages and disadvantages under a number of data scenarios.

\section{Abbreviations}

HTA: Health technology assessment; RCT: Randomised controlled trial; MCMC: Markov chain Monte Carlo; NMA: Network meta-analysis; BP: Begg \& Pilote; BPbias: Begg \& Pilote method with bias-adjustment; BPrandom: Begg \& Pilote method with random effects; BGLMM: Bivariate generalised linear mixed effects model; HPP: Hierarchical power prior; HCP: Hierarchical commensurate prior; MSE: Mean square error; CrlL: Credible interval length; S: Scenario; RA: Rheumatoid arthritis; bDMARDs: Biologic disease-modifying anti-rheumatic drugs; ADA: Adalimumab; ETN: Etanercept; IFX: Infliximab; ABT: Abatacept; RTX: Rituximab; ACR: American College of Rheumatology; REMA: Random effects meta-analysis; FEMA: Fixed effects meta-analysis; DIC: Deviance information criterion; IPD: Individual participant data.

\section{Supplementary Information}

The online version contains supplementary material available at https://doi.org/10.1186/s12874-021-01301-1.

Additional file 1: Supplementary material for: Incorporating single-arm studies in meta-analysis of randomised controlled trials: A simulation

\section{Acknowledgements}

This research used the ALICE/SPECTRE High Performance Computing Facility at the University of Leicester.

\section{Authors' contributions}

JS undertook the data curation and formal analysis, SB and KRA conceptualised the simulation study and provided supervision for JS. All authors read and approved the final manuscript.

\section{Funding}

JS was supported by UK National Institute for Health Research (NIHR) Methods Fellowship [award no. RM-FI-2017-08-027] and NIHR Doctoral Research Fellowship [award no. NIHR300190]. KRA and SB were supported by the UK Medical Research Council [grant no. MR/R025223/1]. KRA is partially supported by Health Data Research (HDR) UK, the NIHR Applied Research Collaboration East Midlands (ARC EM), and as a NIHR Senior Investigator Emeritus (NF-SI-0512-10159). The views expressed are those of the author(s) and not necessarily those of the NIHR or the Department of Health and Social Care.

\section{Availability of data and materials}

All data generated or analysed during this study are included in this published article [and its Supplementary information files].

\section{Declarations}

Ethics approval and consent to participate

Not applicable.

\section{Consent for publication}

Not applicable.

\section{Competing interests}

JS does not have conflict of interest.

SB has served as a paid consultant, providing methodological advice, to NICE and Roche, and has received research funding from European Federation of Pharmaceutical Industries \& Associations (EFPIA) and Johnson \& Johnson. KRA has served as a paid consultant, providing methodological advice, to; Abbvie, Amaris, Allergan, Astellas, AstraZeneca, Boehringer Ingelheim, Bristol-Meyers Squibb, Creativ-Ceutical, GSK, ICON/Oxford Outcomes, Ipsen, Janssen, Eli Lilly, Merck, NICE, Novartis, NovoNordisk, Pfizer, PRMA, Roche and Takeda, and has received research funding from Association of the British Pharmaceutical Industry (ABPI), European Federation of Pharmaceutical Industries \& Associations (EFPIA), Pfizer, Sanofi and Swiss Precision Diagnostics. He is a Partner and Director of Visible Analytics Limited, a healthcare consultancy company. 


\section{Author details}

${ }^{1}$ Biostatistics Research Group, Department of Health Sciences, University of Leicester, Leicester, UK. ${ }^{2}$ Centre for Health Economics, University of York, York, UK.

Received: 13 November 2020 Accepted: 21 April 2021

Published online: 03 June 2021

\section{References}

1. Higgins JPT, Thomas J, Chandler J, Cumpston M, LiT, Page MJ, Welch VA. Cochrane Handbook for Systematic Reviews of Interventions. 2nd Edition. Chichester: Wiley; 2019.

2. Dias S, Welton NJ, Sutton AJ, Ades A. NICE DSU Technical Support Document 1: Introduction to evidence synthesis for decision making. University of Sheffield, Decision Support Unit. 20111-24.

3. Woolacott N, Corbett M, Jones-Diette J, Hodgson R. Methodological challenges for the evaluation of clinical effectiveness in the context of accelerated regulatory approval: an overview. J Clin Epidemiol. 2017;90: 108-18.

4. Dias S, Welton NJ, Sutton AJ, Ades AE. NICE DSU Technical Support Document 2: A Generalised Linear Modelling Framework for Pairwise and Network Meta-Analysis of Randomised Controlled Trials. 2011. http:// www.nicedsu.org.uk. Last updated September 2016.

5. Begg CB, Pilote L. A model for incorporating historical controls into a meta-analysis. Biometrics. 1991;47(3):899-906.

6. Zhang J, Ko C-W, Nie L, Chen Y, Tiwari R. Bayesian hierarchical methods for meta-analysis combining randomized-controlled and single-arm studies. Stat Methods Med Res. 2019;28(5):1293-310.

7. Schmitz S, Maguire Á., Morris J, Ruggeri K, Haller E, Kuhn I, Leahy J, Homer N, Khan A, Bowden J, et al. The use of single armed observational data to closing the gap in otherwise disconnected evidence networks: a network meta-analysis in multiple myeloma. BMC Med Res Methodol. 2018;18(1):66.

8. Thom HH, Capkun G, Cerulli A, Nixon RM, Howard LS. Network meta-analysis combining individual patient and aggregate data from a mixture of study designs with an application to pulmonary arterial hypertension. BMC Med Res Methodol. 2015;15(1):34.

9. Chakravarty K, McDonald H, Pullar T, Taggart A, Chalmers R, Oliver S, Mooney J, Somerville M, Bosworth A, Kennedy T. BSR/BHPR guideline for disease-modifying anti-rheumatic drug (DMARD) therapy in consultation with the British Association of Dermatologists. Rheumatology. 2008;47(6): 924-5.

10. Aletaha D, Neogi T, Silman AJ, Funovits J, Felson DT, Bingham III CO, Birnbaum NS, Burmester GR, Bykerk VP, Cohen MD, et al. 2010 rheumatoid arthritis classification criteria: an American College of Rheumatology/European League Against Rheumatism collaborative initiative. Arthritis Rheum. 2010;62(9):2569-81.

11. Malottki K, Barton P, Tsourapas A, Uthman A, Liu Z. Adalimumab, etanercept, infliximab, rituximab and abatacept for the treatment of rheumatoid arthritis after the failure of a tumour necrosis factor inhibitor: a systematic review and economic evaluation. Health Technol Assess. 2011;15(14):

12. Morris TP, White IR, Crowther MJ. Using simulation studies to evaluate statistical methods. Stat Med. 2019;38(11):2074-102.

13. Lunn DJ, Thomas A, Best N, Spiegelhalter D. WinBUGS-a Bayesian modelling framework: concepts, structure, and extensibility. Stat Comput. 2000;10(4):325-37.

14. DerSimonian R, Laird N. Meta-analysis in clinical trials. Control Clin Trials. 1986;7(3):177-88

15. Spiegelhalter DJ, Best NG, Carlin BP, Van Der Linde A. Bayesian measures of model complexity and fit. J R Stat Soc Ser B Stat Methodol. 2002;64(4): 583-639.

16. Smith TC, Spiegelhalter DJ, Thomas A. Bayesian approaches to random-effects meta-analysis: a comparative study. Stat Med. 1995;14(24):2685-99.

17. Hong H, Chu H, Zhang J, Carlin BP. A Bayesian missing data framework for generalized multiple outcome mixed treatment comparisons. Res Synth Methods. 2016;7(1):6-22.

18. Dias $S$, Ades A. Absolute or relative effects? arm-based synthesis of trial data. Res Synth Methods. 2016;7(1):23.
19. White IR, Turner RM, Karahalios A, Salanti G. A comparison of arm-based and contrast-based models for network meta-analysis. Stat Med. 2019:38(27):5197-213.

20. Grayling MJ, Dimairo M, Mander AP, Jaki TF. A review of perspectives on the use of randomization in phase II oncology trials. J Natl Cancer Inst. 2019;111(12):1255-62.

21. Sabin T, Matcham J, Bray S, Copas A, Parmar MK. A quantitative process for enhancing end of phase 2 decisions. Stat Biopharm Res. 2014;6(1): $67-77$.

22. Martina R, Jenkins $D$, Bujkiewicz $S$, Dequen $P$, Abrams $K$. The inclusion of real world evidence in clinical development planning. Trials. 2018;19(1): $1-12$.

23. Faria R, Hernadez Alava M, Manca A, Wailoo AJ. NICE DSU Technical Support Document 17: The use of observational data to inform estimates of treatment effectiveness for Technology Appraisal: Methods for comparative individual patient data. 2015. p. 19-20. http://www.nicedsu. org.uk.

24. Phillippo DM, Ades AE, Dias S, Palmer S, Abrams KR, Welton NJ. NICE DSU Technical Support Document 18: Methods for population-adjusted indirect comparisons in submission to NICE. 2016. http://www.nicedsu. org.uk.

\section{Publisher's Note}

Springer Nature remains neutral with regard to jurisdictional claims in published maps and institutional affiliations.

Ready to submit your research? Choose BMC and benefit from

- fast, convenient online submission

- thorough peer review by experienced researchers in your field

- rapid publication on acceptance

- support for research data, including large and complex data types

- gold Open Access which fosters wider collaboration and increased citations

- maximum visibility for your research: over $100 \mathrm{M}$ website views per year

At BMC, research is always in progress.

Learn more biomedcentral.com/submissions 